\title{
Electroacupuncture Attenuates Visceral Pain and Reverses Upregulation of TRPV1 Expression in Adult Rats with Neonatal Maternal Deprivation
}

\author{
Hongyan Zhu ${ }^{1 *}$, Shufen $\mathrm{Hu}^{2 *}$, Xiuhua Miao1, Ying Xiao², Guangyin Xu',2\# \\ ${ }^{1}$ Center for Translation Medicine, the Affiliated Zhangjiagang Hospital of Soochow University, \\ Zhangjiagang, China \\ ${ }^{2}$ Jiangsu Key Laboratory of Translational Research and Therapy for Neuro-Psycho-Diseases, Institute of \\ Neuroscience, Soochow University, Suzhou, China \\ Email: "guangyinxu@suda.edu.cn
}

Received 27 December 2015; accepted 6 March 2016; published 9 March 2016

Copyright $@ 2016$ by authors and Scientific Research Publishing Inc.

This work is licensed under the Creative Commons Attribution International License (CC BY). http://creativecommons.org/licenses/by/4.0/

(c) (i) Open Access

\begin{abstract}
Irritable bowel syndrome (IBS) is characterized by chronic visceral hypersensitivity that companied by altered bowel movement. However, the treatment options are very limited. The aim of this study was to investigate effects of electroacupuncture (EA) on visceral hypersensitivity in a rat model of IBS and to explore the underlying mechanisms of EA effects. Visceral hypersensitivity was established by neonatal maternal deprivation (NMD) in male rats on postnatal days 2 - 15. Behavioral experiments were conducted at the age of 7 weeks. Treatment with EA at Zusanli (stomach-36, ST-36) significantly reduced abdominal withdrawal reflex (AWR) scores in NMD rats but not in age-matched healthy control rats. In addition, EA treatment hyperpolarized resting membrane potentials, increased the rheobase and reduced the numbers of action potentials evoked by 2 and 3 times rheobase current stimulation of dorsal root ganglion (DRG) neurons innervating the colon. NMD markedly enhanced expression of TRPV1 in colon related DRGs while EA treatment drastically suppressed the expression of TRPV1 in DRGs of NMD rats. These data suggest that EA treatment produced an analgesic effect, which might be mediated at least in a part by suppression of TRPV1 expression and by inhibition of neuronal excitability of primary sensory neurons in rats with chronic visceral pain.
\end{abstract}

*These authors contribute to this work equally.

\#Corresponding author.

How to cite this paper: Zhu, H.Y., Hu, S.F., Miao, X.H., Xiao, Y. and Xu, G.Y. (2016) Electroacupuncture Attenuates Visceral Pain and Reverses Upregulation of TRPV1 Expression in Adult Rats with Neonatal Maternal Deprivation. Chinese Medicine, 7, 1-9. http://dx.doi.org/10.4236/cm.2016.71001 


\title{
Keywords
}

\author{
Electroacupuncture, Irritable Bowel Syndrome, Chronic Visceral Pain, Dorsal Root Ganglion, \\ TRPV1
}

\section{Introduction}

Irritable bowel syndrome (IBS) is one of the most common functional gastrointestinal disorders, featured by chronic visceral hypersensitivity $(\mathrm{CVH})$ and altered bowel movement. The treatment of CVH in IBS patients remains challenging for the clinicians. Both acupuncture and electroacupuncture (EA) have been applied in treating many diseases including chronic pain for the past several decades [1]-[3]. It has been demonstrated that EA treatment produces a significant improvement both in general well-being and in symptoms of chronic visceral hypersensitivity [4]. Although these data suggest that EA could be a promising method to treat chronic pain in patients, scientific evidence is very little and frequently contradictory. Thus, further observations of the efficacy and the underlying mechanisms of EA are definitely needed.

We have previously reported that EA treatment significantly inhibited the neuronal excitability of dorsal root ganglion (DRG) neurons and attenuated the CVH in a rat model of IBS induced by neonatal colonic inflammation [5]. The sensitization of DRGs is considered to be a major cause of aberrant pain induced by neonatal maternal deprivation (NMD) [6] [7]. However, whether EA produced an inhibitory effect on the hyperexcitability of DRG neurons of adult rats with NMD is not clear. Emerging evidence has suggested that TRPV1 played an important role in inflammatory pain [8] and neuropathic pain [9]. A growing body of evidence suggests a pronociceptive role for TRPV1 in IBS [10] [11]. In a painful IBS model induced by neonatal colonic inflammation, TRPV1 expression is significantly increased in DRGs of rats [5]. However, roles of TRPV1 signaling pathway in NMD-induced visceral pain remain largely unknown. It would be of considerable interest to explore whether EA exerts its therapeutic effect via the inhibition of TRPV1 in DRGs of adult rats with NMD.

We therefore hypothesized that TRPV1 activation and sensitization of DRG neurons participated in NMDinduced visceral hypersensitivity and that EA treatment produced analgesic effects via suppression of TRPV1 expression in DRGs. To test this hypothesis, we investigated roles of TRPV1 and excitability of DRG neurons in adult NMD rats with EA treatment. Our results indicate that NMD-induced visceral hypersensitivity is likely mediated by upregulation of TRPV1 expression and an increase of cell excitability in DRG neurons, and that EA treatment relieves visceral hypersensitivity through the inhibition of TRPV1 upregulation and the reduction of excitability of DRG neurons innervating the colon in this setting.

\section{Materials and Methods}

\subsection{Induction of Chronic Visceral Pain}

Experiments were conducted on adult male Sprague-Dawley (SD) rats. Handling and care of rats were approved by the Institutional Animal Care and Use Committee of Soochow University and were in accordance with the guidelines of the International Association for the Study of Pain. As described previously, CVH was established by neonatal maternal deprivation (NMD) [12] [13]. Briefly, separating pups from the maternity cage and placed in isolated cages were performed 3 hours daily from postnatal day (PND) 2 to 15 . The inside temperature of the isolated cages was kept around $32^{\circ} \mathrm{C}$ by using an electric blanket. After the separation, pups were returned to their dams. Pups in the control (CON) group were not exposed to handling and were maintained in their maternity cage with the dam. Only male pups were used in the present study. Experiments were performed at 7 weeks of age in a total of 38 rats (CON: $n=6$ for behavior test, $n=7$ for patch clamp recordings, $n=6$ for western blot analysis; NMD: $\mathrm{n}=6$ for behavior test, $\mathrm{n}=7$ for patch clamp recordings, $\mathrm{n}=6$ for western blot analysis).

\subsection{Behavioral Studies to Measure Nocifensive Responses to Graded CRD}

$\mathrm{CVH}$ was determined by assessing the behavioral responses to colorectal distention (CRD) at the age of 7 weeks as described previously [14] [15]. Under mild sedation by $1 \%$ Brevital (25 mg/kg, i.p.), a flexible latex balloon $(6 \mathrm{~cm})$ attached to a tygon tubing was inserted $8 \mathrm{~cm}$ into the descending colon and rectum via the anus. The 
plastic tube was kept in place by taping the tubing to the rat tail. The rat was positioned in small Lucite cubicles for $30 \mathrm{~min}$ to adapt. CRD was determined by quickly inflating the balloon to a constant pressure using a sphygmomanometer. The balloon was inflated to 20, 40, 60 and $80 \mathrm{mmHg}$, for $20 \mathrm{~s}$ followed by $2 \mathrm{~min}$ rest. Behavioral responses to CRD were determined by visual observation of the abdominal withdrawal reflex (AWR) by an observer in a blinded manner. The AWR score was assigned as follows: 0 with normal behavior, 1 with slight head movement without abdominal response, 2 with contraction of abdominal muscles, 3 with lifting of abdominal wall or 4 with body arching and lifting of pelvic structures.

\subsection{Electroacupuncture Treatment}

Electroacupuncture (EA) treatment was given at ST-36 (stomach-36, Zusanli) of control and NMD rats [1] [4]. A pair of stainless steel suturehook-shaped needles were inserted a depth of $5 \mathrm{~mm}$ into the skin and underlying muscles of bilateral ST-36. Stimulation was delivered through the needles inserted into the acupoints by an EA apparatus (Model G-6805-2; Shanghai Medical Electronic Apparatus Company, Shanghai, China) with a constant rectangular current of alternating trains of dense-sparse frequencies $(100 \mathrm{~Hz}$ for 1.05 seconds and $2 \mathrm{~Hz}$ for 2.85 seconds alternately, pulse width, $0.1 \mathrm{~ms}$ ). These parameters have been reported as the most optimum condition to induce maximal release of metenkephalin and dynorphin A. Electrical stimulus intensity was determined as the threshold for a detectable muscle twitch (approximately $1 \mathrm{~mA}$ ). The stimulation was applied for $30 \mathrm{mi}-$ nutes once to observe the acute effect or 30 minutes once per day for consecutive 5 days to observe the accumulative effect in these rats.

\subsection{Cell Labeling}

DRG neurons innervating the colon were labeled by injection of 1, 1'-dioleyl-3, 3, 3', 3-tetramethylindocarbocyanine methanesulfonate (DiI, Invitrogen) into the colon wall before patch clamp recordings. Briefly, adult rats ( $\sim 6$ weeks old) were anaesthetized by a mixture of ketamine ( $80 \mathrm{mg} / \mathrm{kg}$, i. p.) and xylazine (5 - $10 \mathrm{mg} / \mathrm{kg}, \mathrm{i}$. p.). Surgery was performed to expose the colon. DiI was injected in $\sim 1 \mu \mathrm{l}$ volume at 10 sites on the exposed colon extending from the level of the bladder to $\sim 6 \mathrm{~cm}$ in an oral direction [16]. The needle was left in place for $1 \mathrm{~min}$ to prevent DiI leakage. After surgery, these rats were returned to their house and were free access to drinking water and standard food pellets.

\subsection{Dissociation of DRG Neurons and Patch Clamp Recordings}

NMD ( 7 weeks) or age-matched control rats were euthanized by cervical dislocation 10 days after DiI injection. Bilateral DRGs (T13-L2) were dissected out for patch clamp recording as described previously [13]. In brief, these DRGs were moved to an ice-cold, oxygenated fresh solution, containing (in mM): $130 \mathrm{NaCl}, 5 \mathrm{KCl}, 2$ $\mathrm{KH}_{2} \mathrm{PO}_{2}, 1.5 \mathrm{CaCl}_{2}, 6 \mathrm{MgSO}_{2}, 10$ glucose and 10 HEPES, pH 7.2 (Osmolarity: 305 mOsm). The dissecting solution also included collagenase D (1.8 - $2.0 \mathrm{mg} / \mathrm{ml}$; Roche, Indianapolis, Indiana, USA) and trypsin (1.2 - 1.5 $\mathrm{mg} / \mathrm{ml}$; Sigma, St Louis, Missouri, USA). After incubation in this solution for $1.5 \mathrm{~h}$ at $34.5^{\circ} \mathrm{C}$, DRGs were then taken out from the enzyme solution, washed and moved to $0.5 \mathrm{ml}$ of the dissecting solution containing DNase $(0.5 \mathrm{mg} / \mathrm{ml}$; Sigma, St Louis, Missouri, USA). A single cell suspension was then formed by repeated trituration through flame-polished glass pipettes. The normal external solution included (in $\mathrm{mM}$ ): $130 \mathrm{NaCl}, 5 \mathrm{KCl}, 2$ $\mathrm{KH}_{2} \mathrm{PO}_{4}, 2.5 \mathrm{CaCl}_{2}, 1 \mathrm{MgCl}_{2}$, 10 HEPES, 10 glucose, with $\mathrm{pH}$ adjusted to 7.2 with $\mathrm{NaOH}$, Osmolarity: 295 $300 \mathrm{mOsm}$. DiI-labeled neurons were selected by their fluorescence under the fluorescent microscope (Olympus IX71) for the patch-clamp recordings. Recording pipettes were pulled from borosilicate glass tubing using a horizontal puller (P-97, Sutter Instruments). Patch pipettes had a resistance of 4 - $7 \mathrm{M} \Omega$ when they were filled with the pipette solution containing (in $\mathrm{mM}$ ): 140 potassium gluconate, $10 \mathrm{NaCl}, 10 \mathrm{HEPES}, 10$ glucose, 5 EGTA and $1 \mathrm{CaCl}_{2}, \mathrm{pH}=7.25$ adjusted with $\mathrm{KOH}$; Osmolarity = $292 \mathrm{mOsm}$. Resting potential (RP) and action potentials (APs) evoked by current stimulation were recorded by an HEKA EPC10 patch clamp amplifier (HEKA; Germany). Electrophysiological recordings were performed at room temperature of $\sim 22^{\circ} \mathrm{C}$. Data were saved in a personal computer for a later analysis using FitMaster (HEKA; Germany).

\subsection{Western Blot Experiments}

The protein expression of TRPV1 from colon DRGs (T13-L2) of control, NMD and NMD + EA rats was deter- 
mined by western blotting, as described previously [13]. The primary antibodies were rabbit anti-TRPV1 (1:1000) and mouse anti- $\beta$-actin (1:1000). $\beta$-actin was used as a loading control. All protein samples were normalized to $\beta$-actin.

\subsection{Data Analysis}

Data are shown as mean \pm SD. Statistical analysis were determined using software including OriginPro 8 (OriginLab, US) and Matlab (Mathworks, US). Normality of all data was first examined before further analyses. Significance of all data was assessed using Wilcoxon rank sum test. The level of significance was set at $\mathrm{p}<$ 0.05 .

\section{Results}

\subsection{EA Treatment Attenuates AWRs in NMD Rats}

To determine whether EA suppressed visceral hypersensitivity of NMD rats, CRD was applied to examine the behavior responses of NMD rats before (PRE) and after EA treatment. Compared with PRE-EA, EA treatment for 30 minutes markedly suppressed the AWRs at 0 and 30 min (Figure 1 (a), ${ }^{*} \mathrm{p}<0.05, \mathrm{n}=6$, Wilcoxon rank sum test). We next determined whether the same EA treatment also plays an effect on age-matched healthy control rats. EA treatment did not have any effect on AWRs in control rats when compared with PRE EA treatment (Figure 1(b), $n=6$ ). These results suggest that EA treatment plays a therapeutic effect on visceral hypersensitivity of NMD rats.
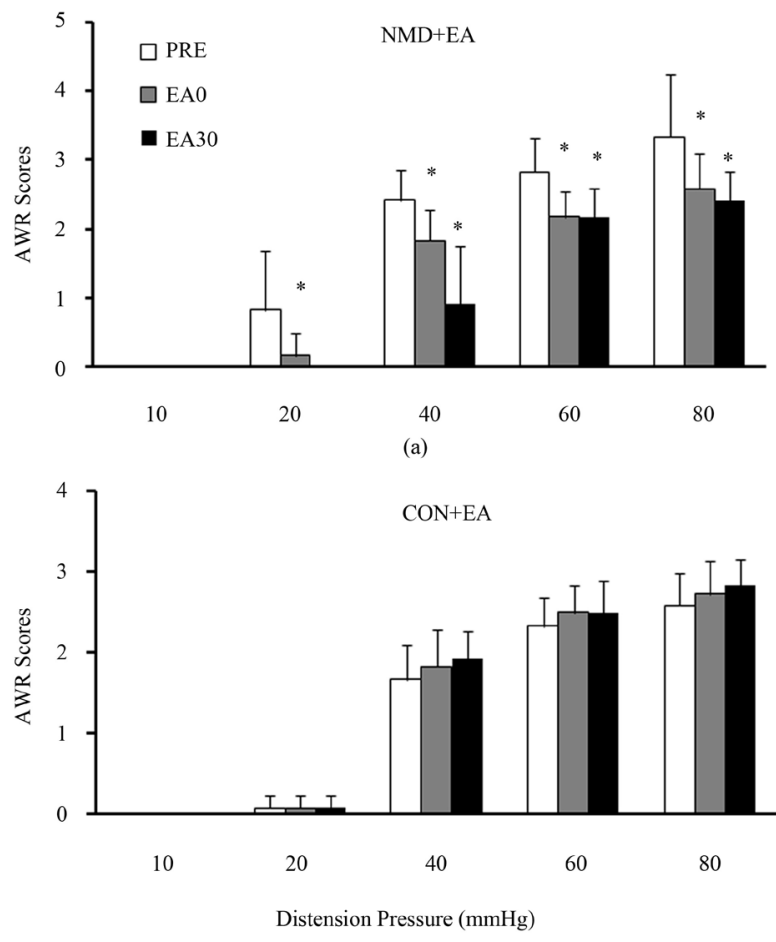

(b)

Figure 1. EA treatment attenuated abdominal withdrawal reflex (AWR) scores. (a) Time course of effect of one time EA treatment (30 min) on NMD rats. EA at ST-36 produced the analgesic effect in NMD rats when compared with PRE. This effect lasted for about 30 minutes within our observation time period. $n=6$ rats, ${ }^{*} p<0.05$. (b) Time course of effect of EA treatment on control rats. The EA treatment had no effect on AWRs in age-matched healthy control rats. $n=6$ rats, $p>0.05$ when compared with PRE. 


\subsection{EA Treatment Inhibits Neuronal Hyperexcitability of DRG Neurons Innervating the Colon}

Since EA treatment attenuated the AWRs in NMD rats, we next investigated whether this therapeutic effect is mediated by suppression of excitability of colon neurons. To determine the effect of EA on neuronal excitability, patch clamp recordings were performed on colon specific DRG neurons that were labeled by DiI (Figure 2(a) and Figure 2(b), arrow). EA treatment significantly hyperpolarized resting membrane potentials (RP, Figure 2(c)). The RPs were $-49.71 \pm 4.3(n=28$ cells $),-45.24 \pm 3.47(n=41$ cells) and $-47.15 \pm 3.46(n=13$ cells) for CON, NMD and EA, respectively. In addition, EA treatment dramatically enhanced rheobase when compared with the NMD group (Figure 2(d), CON: $0.24 \pm 0.11 \mathrm{nA}, \mathrm{n}=28$; NMD: $0.17 \pm 0.09 \mathrm{nA}, \mathrm{n}=41$; EA: $0.3 \pm 0.1$ $\mathrm{nA}, \mathrm{n}=13, \stackrel{* *}{\mathrm{p}}<0.01$, compared with CON; ${ }_{\mathrm{p}}<0.05$, compared with NMD. Wilcoxon rank sum test). EA treatment markedly decreased the number of action potentials (APs) evoked by $2 \mathrm{X}$ and $3 \mathrm{X}$ rheobase current stimulation (Figures 3(a)-(c)). The average numbers of APs evoked by 2X rheobase stimulation were $1.05 \pm$ 0.49 for CON ( $n=28$ cells), $2.64 \pm 3.01$ for NMD ( $n=41$ cells) and $1.23 \pm 0.44$ for EA ( $n=13$ cells), respectively (Figure 3(b), ${ }^{*} \mathrm{p}<0.05$, compared with CON; ${ }^{*} \mathrm{p}<0.05$, compared with NMD. Wilcoxon rank sum test). The average numbers of APs evoked by $3 \mathrm{X}$ rheobase stimulation were $1.96 \pm 1.6$ for CON ( $\mathrm{n}=28$ cells); $3.5 \pm$ 3.01 for NMD ( $\mathrm{n}=41$ cells) and $1.00 \pm 0.00$ for EA ( $\mathrm{n}=13$ cells), respectively (Figure 3(c), ${ }^{*} \mathrm{p}<0.05$, compared with CON; ${ }^{\#} \mathrm{p}<0.05$, compared with NMD. Wilcoxon rank sum test).

Additional membrane properties of DRG neurons innervating the colon were also examined and the results were shown in Table 1. Neither NMD nor EA treatment altered the cell size, input resistance, AP threshold, amplitude, overshoot, duration and latency of DRG neurons innervating the colon (Table 1).

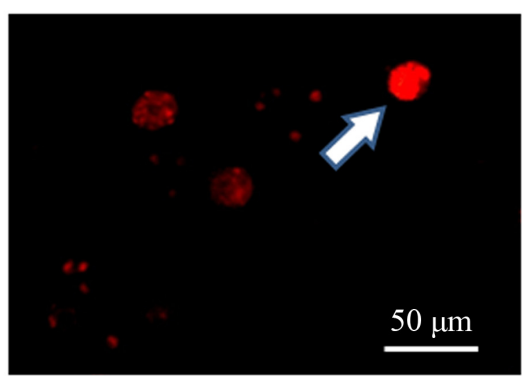

(a)

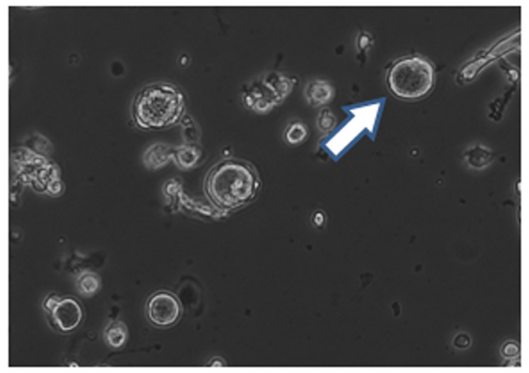

(b)

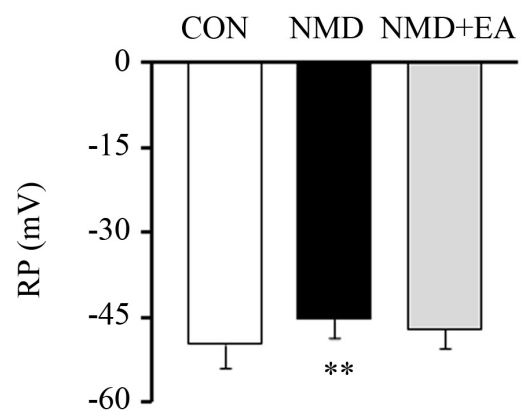

(c)

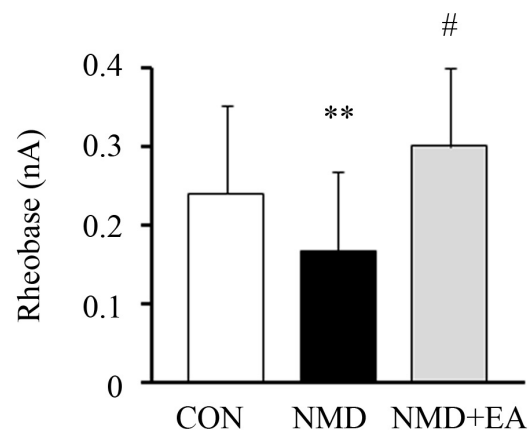

(d)

Figure 2. EA treatment hyperpolarized resting membrane potentials and enhanced rheobase. (a) DiI-fluorescence (upper) and (b) bright-field (lower) images of DRG neurons. A colon innervating DRG neuron is shown in red (arrow). Bar $=50 \mu \mathrm{m}$. (c) Bar graph showed NMD significantly depolarized rest membrane potential (RP) while EA treatment reversed the depolarization of RP. $n=7$ rats for each group, ${ }^{* *} \mathrm{p}<0.01$, compared with CON. (d) Bar graph showed NMD markedly decreased rheobase of current stimulation of colon specific DRG neuron while EA treatment increased rheobase of current stimulation of colon specific DRG neuron. $n=7$ rats for each group, ${ }^{* *} \mathrm{p}<0.01$, compared with CON; ${ }^{*} \mathrm{p}<0.05$, compared with NMD. 


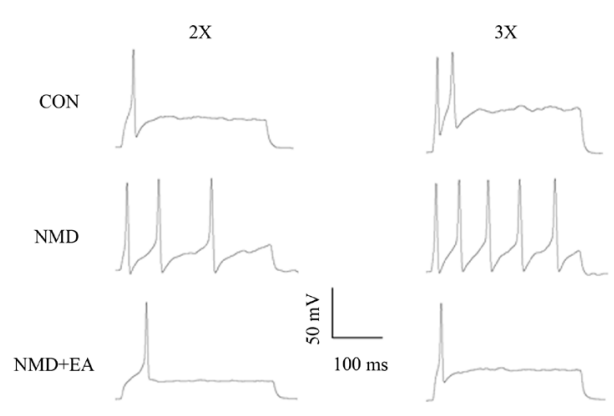

(a)

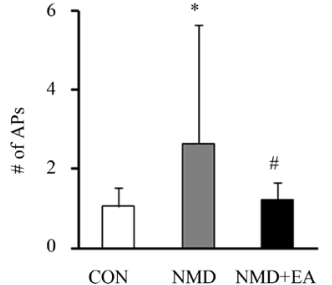

(b)

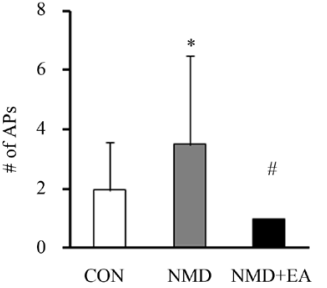

(c)

Figure 3. EA treatment reduced numbers of action potentials (APs) evoked by current stimulation. (a) Examples of APs by $2 \times$ and $3 \times$ rheobase current injection for control group (CON, top), NMD group (NMD, middle) and electroacupuncture group (NMD + EA, bottom). (b) Bar graph showed NMD significantly increased number of APs evoked by $2 \times$ rheobase current stimulation while EA treatment reversed NMD-induced increase in number of APs elicited by $2 \times$ rheobase current stimulation. $\mathrm{n}=7$ rats for each group, ${ }^{*} \mathrm{p}<0.05$, compared with CON; ${ }^{*} \mathrm{p}<0.05$ compared with NMD. (c) Bar graph showed NMD greatly increased number of APs evoked by $3 \times$ rheobase current stimulation while EA treatment reversed NMD-induced increase in number of APs elicited by $3 \times$ rheobase current stimulation. $n=7$ rats for each group, ${ }^{*} \mathrm{p}<0.05$, compared with CON; ${ }^{\mathrm{p}}<0.05$ compared with NMD.

Table 1. Membrane characteristics of colon-specific DRG neurons in control (CON), NMD and NMD+EA-treated rats.

\begin{tabular}{|c|c|c|c|}
\hline & CON & NMD & NMD + EA \\
\hline Cell Size $(\mu \mathrm{m})$ & $\begin{array}{c}27.8 \pm 2.8 \\
(n=28)\end{array}$ & $\begin{array}{c}26.6 \pm 2.35 \\
(\mathrm{n}=28)\end{array}$ & $\begin{array}{c}29.4 \pm 2.08 \\
(\mathrm{n}=13)\end{array}$ \\
\hline $\operatorname{Rin}(\mathrm{M} \Omega)$ & $\begin{array}{c}674.0 \pm 251.3 \\
(n=28)\end{array}$ & $\begin{array}{c}703.5 \pm 199.3 \\
(n=28)\end{array}$ & $\begin{array}{c}560.4 \pm 217.89 \\
(\mathrm{n}=13)\end{array}$ \\
\hline $\mathrm{RP}(\mathrm{mV})$ & $\begin{array}{c}-49.7 \pm 4.3 \\
(\mathrm{n}=28)\end{array}$ & $\begin{aligned} &-45.2 \pm 3.47^{* * *} \\
&(\mathrm{n}=28)\end{aligned}$ & $\begin{array}{c}-47.2 \pm 3.46 \\
(n=13)\end{array}$ \\
\hline Rheobase (nA) & $\begin{array}{l}0.24 \pm 0.11 \\
(\mathrm{n}=28)\end{array}$ & $\begin{array}{c}0.17 \pm 0.09^{* *} \\
(\mathrm{n}=28)\end{array}$ & $\begin{array}{l}0.30 \pm 0.1^{\#} \\
(\mathrm{n}=13)\end{array}$ \\
\hline \# of AP (2xrheobase) & $\begin{array}{l}1.0 \pm 0.4 \\
(n=25)\end{array}$ & $\begin{array}{l}2.6 \pm 3^{*} \\
(\mathrm{n}=27)\end{array}$ & $\begin{array}{c}1.2 \pm 0.44^{\#} \\
(\mathrm{n}=13)\end{array}$ \\
\hline \#of AP (3xrheobase) & $\begin{array}{l}1.0 \pm 1.6 \\
(\mathrm{n}=21)\end{array}$ & $\begin{array}{l}3.5 \pm 3^{*} \\
(\mathrm{n}=25)\end{array}$ & $\begin{array}{c}1.0 \pm 0.0^{\#} \\
(\mathrm{n}=8)\end{array}$ \\
\hline AP Threshold (mV) & $\begin{array}{c}-16.8 \pm 10.69 \\
(\mathrm{n}=28)\end{array}$ & $\begin{array}{c}-18.4 \pm 7.22 \\
(\mathrm{n}=28)\end{array}$ & $\begin{array}{c}-16.3 \pm 4.25 \\
(\mathrm{n}=13)\end{array}$ \\
\hline AP Amplitude (mV) & $\begin{array}{c}75.7 \pm 14.68 \\
(\mathrm{n}=28)\end{array}$ & $\begin{array}{c}71.0 \pm 13.66 \\
(\mathrm{n}=28)\end{array}$ & $\begin{array}{c}79.3 \pm 9.93 \\
(\mathrm{n}=13)\end{array}$ \\
\hline AP Overshoot (mV) & $\begin{array}{c}26.0 \pm 13.41 \\
(\mathrm{n}=28)\end{array}$ & $\begin{array}{c}25.8 \pm 12.08 \\
(\mathrm{n}=28)\end{array}$ & $\begin{array}{c}32.2 \pm 8.86 \\
(\mathrm{n}=13)\end{array}$ \\
\hline AP Duration (ms) & $\begin{array}{l}3.3 \pm 1.0 \\
(\mathrm{n}=28)\end{array}$ & $\begin{array}{c}3.3 \pm 1.06 \\
(\mathrm{n}=28)\end{array}$ & $\begin{array}{c}2.9 \pm 0.49 \\
(\mathrm{n}=13)\end{array}$ \\
\hline Latency (ms) & $\begin{array}{c}72.8 \pm 101.92 \\
(\mathrm{n}=28)\end{array}$ & $\begin{array}{c}55.1 \pm 90.4 \\
(\mathrm{n}=28)\end{array}$ & $\begin{array}{c}50.9 \pm 91.57 \\
(\mathrm{n}=13)\end{array}$ \\
\hline
\end{tabular}

Values are mean $\pm \mathrm{SD}$, with sample size in parenthesis; Rin, Input resistance; RP, Resting membrane potential; AP, Action potential. ${ }^{*} \mathrm{p}<0.05$, ${ }^{* * *} \mathrm{p}<$ $0.01,{ }^{* * *} \mathrm{p}<0.001$, compared with CON; ${ }^{*} \mathrm{p}<0.05$, compared with NMD. 


\subsection{EA Treatment Suppresses TRPV1 Expression in DRGs of NMD Rats}

To determine the expression of TRPV1 in DRGs of NMD rats, proteins isolated from both sides of T13-L2 DRGs of NMD and age-matched control rats were probed with anti-TRPV1 antibody. Anti-TRPV1 antibody labeled a $90 \mathrm{Kd}$ molecular weight protein. Seven weeks after NMD, the relative densitometry of TRPV1 was $0.29 \pm$ $0.087(\mathrm{n}=3)$ and $0.56 \pm 0.21(\mathrm{n}=3)$ for control $(\mathrm{CON})$ and NMD, respectively. The levels of expression of TRPV1 were significantly increased after NMD (Figure 4(a), ${ }^{*} \mathrm{p}<0.05$, Wilcoxon rank sum test). EA treatment was administrated at age of 6 weeks once daily for consecutive 5 days. The relative densitometry was $0.91 \pm$ $0.05(n=3)$ and $0.59 \pm 0.33(n=3)$ for NMD and EA, respectively. EA treatment significantly reduced expression of TRPV1 (Figure $4(\mathrm{~b}),{ }^{*} \mathrm{p}<0.05$, Wilcoxon rank sum test). Thus, EA treatment reverses the upregulation of TRPV1 expression in colon DRGs isolated from NMD rats.

\section{Discussion}

In the present study we provided evidence to support our hypothesis that electroacupuncture at acupoints of ST-36 (stomach-36, Zusanli) relieved visceral hypersensitivity in adult rats with neonatal maternal deprivation, as indicated by the inhibitory effects of EA on AWR scores. In addition, our present study demonstrated that EA treatment suppressed TRPV1 expression and reduced cell excitability of DRG neurons innervating the colon, which might be the mechanism by which EA treatment produced analgesic effects on the visceral hypersensitivity. These findings further support the previous reports and provide additional evidence for EA treatment for visceral pain in a rat model of IBS [4] [17].

NMD-induced rat model of visceral pain is a frequently used animal model for study mechanisms of visceral pain. After EA treatment, NMD rats showed a significant reduction in visceromoter responses to colorectal distention while the same EA treatment did alter the AWRs in age-matched healthy control rats. This suggested that EA-produced analgesic effect is not a non-specific effect and most likely is disease-related, further confirming the specificity of EA effects. It seems that the analgesic effect induced by EA in various conditions may be mediated by different mechanisms depending on the specific conditions [12] [18]. We have previously reported that EA treatment inhibited cell excitability of DRG neurons in rats with neonatal colonic inflammation, another rat model of IBS [4]. In the present study, we further confirmed that EA reversed the hyperexcitability of DRG neurons innervating the colon. Moreover, the present findings show that TRPV1 expression was greatly upregulated in colon DRGs and that EA treatment reversed the up regulation of TRPV1 expression in NMD rats. To the best of our knowledge, this is the first report showing an involvement of electroacupuncture in the primary sensory neurons in a painful NMD rat model and inhibition of this pathway underlies the EA-induced analgesic effect in visceral hypersensitivity in this study.

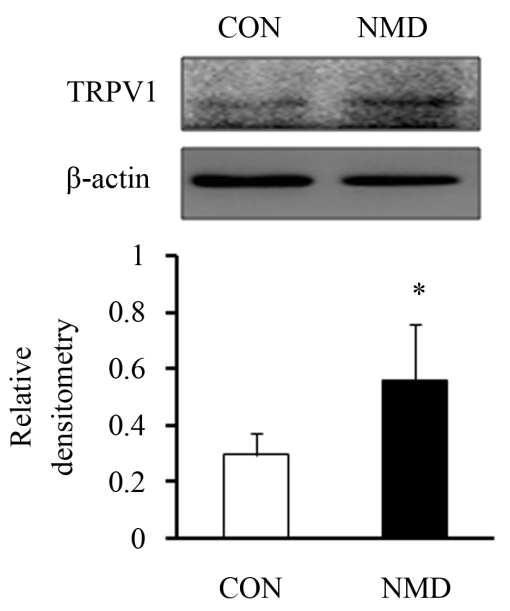

(a)

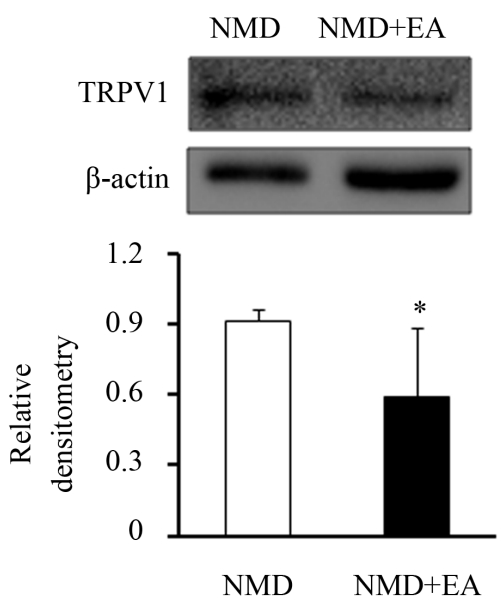

(b)

Figure 4. EA treatment suppressed TRPV1 expression. (a) NMD significantly increased TRPV1 expression of colon DRGs when compared with age-matched control group. $\mathrm{n}=$ 3 rats for each group, ${ }^{*} \mathrm{p}<0.05$. (b) EA treatment markedly decreased TRPV1 expression when compared with NMD group. $\mathrm{n}=3$ rats for each group, $\stackrel{*}{\mathrm{p}}<0.05$. 
One of the limitations of the present study is the use of male rats. The purpose of only using male rats is to exclude the possible effects of estrogenon female ones [19] [20]. The female hormones may induce visceral pain periodically compared with male ones although the prevalence of visceral pain is higher in female patients than in male ones. Another limitation is that only ST-36 acupoints were used in the present study. The reason why ST-36 was used is because we have previously showed that ST-36 is an effective acupoint in a rat model of IBS induced by neonatal colonic inflammation [4] and by chronic heterotypical stress as well [1]. Future studies are warranted to investigate the roles of female hormones and other acupoints in this model of visceral pain.

\section{Conclusion}

In conclusion, the present study demonstrated that NMD led to upregulation of TRPV1 expression and hyperexcitability of DRG neurons innervating the colon. EA treatment at ST-36 significantly attenuates the visceral hypersensitivity in association with reduced expression of TRPV1 and inhibition of colon specific DRG neurons in NMD rats.

\section{Acknowledgements}

This work was supported by grants from National Natural Science Foundation of China (81230024 and 81471137 to GYX and 81500952 to SFH) and from the Priority Academic Program Development of Jiangsu Higher Education Institutions of China. This project was subject to the second affiliated hospital of Soochow university preponderant clinic discipline group project funding (XKQ2015008). The funders had no role in the study design, data collection and analysis, decision to publish, or preparation of the manuscript.

\section{Disclosure}

The authors declare no conflict of interest.

\section{References}

[1] Tu, W.Z., Cheng, R.D., Cheng, B., et al. (2012) Analgesic Effect of Electroacupuncture on Chronic Neuropathic Pain Mediated by P2X3 Receptors in Rat Dorsal Root Ganglion Neurons. Neurochemistry International, 60, 379-386. http://dx.doi.org/10.1016/j.neuint.2012.01.006

[2] Yin, J. and Chen, J.D. (2010) Gastrointestinal Motility Disorders and Acupuncture. Autonomic Neuroscience, 157, 3137. http://dx.doi.org/10.1016/j.autneu.2010.03.007

[3] Zhao, Z.Q. (2008) Neural Mechanism Underlying Acupuncture Analgesia. Progress in Neurobiology, 85, 355-375. http://dx.doi.org/10.1016/j.pneurobio.2008.05.004

[4] Xu, G.Y., Winston, J.H. and Chen, J.D. (2009) Electroacupuncture Attenuates Visceral Hyperalgesia and Inhibits the Enhanced Excitability of Colon Specific Sensory Neurons in a Rat Model of Irritable Bowel Syndrome. Neurogastroenterology \& Motility, 21, 1302-e125. http://dx.doi.org/10.1111/j.1365-2982.2009.01354.x

[5] Winston, J., Shenoy, M., Medley, D., et al. (2007) The Vanilloid Receptor Initiates and Maintains Colonic Hypersensitivity Induced by Neonatal Colon Irritation in Rats. Gastroenterology, 132, 615-627. http://dx.doi.org/10.1053/j.gastro.2006.11.014

[6] Li, L., Xie, R., Hu, S., et al. (2012) Upregulation of CBS Expression by Nuclear Factor-Kappa B Activation Contributes to Visceral Hypersensitivity in Adult Rats with Neonatal Maternal Deprivation. Molecular Pain, 8, 89.

[7] Hu, S., Xiao, Y., Zhu, L.Y., et al. (2013) Neonatal Maternal Deprivation Sensitizes Voltage-Gated Sodium Channel Currents in Colon-Specific Dorsal Root Ganglion Neurons in Rats. American Journal of Physiology Gastrointestinal and Liver Physiology, 304, G311-G321. http://dx.doi.org/10.1152/ajpgi.00338.2012

[8] Schwartz, E.S., La, J.-H., Scheff1, N.N., et al. (2013) TRPV1 and TRPA1 Antagonists Prevent the Transition of Acute to Chronic Inflammation and Pain in Chronic Pancreatitis. The Journal of Neuroscience, 33, 5603-5611. http://dx.doi.org/10.1523/JNEUROSCI.1806-12.2013

[9] Armero, P., Muriel, C., López, M., et al. (2012) [Analysis of TRPV1 Gene Polymorphisms in Spanish Patients with Neuropathic Pain]. Medicina Clínica, 139, 1-4. http://dx.doi.org/10.1016/j.medcli.2011.10.028

[10] Zhou, Q., Yang, L.Q., Larson, S., et al. (2015) Decreased miR-199 Augments Visceral Pain in Patients with IBS through Translational Upregulation of TRPV1. Gut. http://dx.doi.org/10.1136/gutjnl-2013-306464

[11] Hughes, P.A., Brierley, S.M., Martin, C.M., et al. (2009) TRPV1-Expressing Sensory Fibres and IBS: Links with Im- 
mune Function. Gut, 58, 465-466. http://dx.doi.org/10.1136/gut.2008.161760

[12] Zhang, Y., Meng, X., Li, A., et al. (2012) Electroacupuncture Alleviates Affective Pain in an Inflammatory Pain Rat Model. European Journal of Pain, 16, 170-181. http://dx.doi.org/10.1016/j.ejpain.2011.07.002

[13] Hu, S., Xu, W.Y., Miao, X.H., et al. (2013) Sensitization of Sodium Channels by Cystathionine Beta-Synthetase Activation in Colon Sensory Neurons in Adult Rats with Neonatal Maternal Deprivation. Experimental Neurology, 248, 275-285. http://dx.doi.org/10.1016/j.expneurol.2013.06.027

[14] Al-Chaer, E.D., Kawasaki, M. and Pasricha, P.J. (2000) A New Model of Chronic Visceral Hypersensitivity in Adult Rats Induced by Colon Irritation during Postnatal Development. Gastroenterology, 119, 1276-1285. http://dx.doi.org/10.1053/gast.2000.19576

[15] Tian, S.L., Wang, X.Y. and Ding, G.H. (2008) Repeated Electro-Acupuncture Attenuates Chronic Visceral Hypersensitivity and Spinal Cord NMDA Receptor Phosphorylation in a Rat Irritable Bowel Syndrome Model. Life Sciences, 83, 356-363. http://dx.doi.org/10.1016/j.lfs.2008.06.027

[16] Wang, Y., et al. (2012) Upregulation of Cystathionine Beta-Synthetase Expression Contributes to Visceral Hyperalgesia Induced by Heterotypic Intermittent Stress in Rats. PLOS ONE, 7, e53165. http://dx.doi.org/10.1371/journal.pone.0053165

[17] Xu, K.D., et al. (2010) Effect of Pre-Electroacupuncture on p38 and c-Fos Expression in the Spinal Dorsal Horn of Rats Suffering from Visceral Pain. Chinese Medical Journal, 123, 1176-1181.

[18] Gim, G.T., Lee, J., Park, E., et al. (2011) Electroacupuncture Attenuates Mechanical and Warm Allodynia through Suppression of Spinal Glial Activation in a Rat Model of Neuropathic Pain. Brain Research Bulletin, 86, 403-411. http://dx.doi.org/10.1016/j.brainresbull.2011.09.010

[19] Cao, D.Y., et al. (2015) Epigenetic Upregulation of Metabotropic Glutamate Receptor 2 in the Spinal Cord Attenuates oestrogen-Induced Visceral Hypersensitivity. Gut, 64, 1913-1920. http://dx.doi.org/10.1136/gutjnl-2014-307748

[20] Ji, Y., Bai, G., Cao, D.-Y., et al. (2015) Estradiol Modulates Visceral Hyperalgesia by Increasing Thoracolumbar Spinal GluN2B Subunit Activity in Female Rats. Neurogastroenterology \& Motility, 27, 775-786.

http://dx.doi.org/10.1111/nmo.12549 